\title{
Las obras de arte del Estado y su inmunidad
}

Fecha de recepción: 12 de enero de 2016

Fecha de aceptación: 29 de febrero de 2016

Doi: 10.12804/revistas.urosario.edu.co/acdi/a.5301

\section{Soledad Torrecuadrada García-Lozano*}

Resumen: en los últimos tiempos, la tecnología ha tenido por efecto empequeñecer el mundo en todos los sentidos. Así, ahora no solo las distancias se recorren en menos tiempo, sino además tenemos ocasión de contemplar museos 'satélites' del Museo Hermitage de San Petersburgo en Málaga o Ámsterdam, por ejemplo, o colecciones prestadas por museos a otros para que disfrutemos con ellas en el otro lado del mundo de donde habitualmente se exponen. Con ocasión de estos préstamos y la aplicación de nuevas tecnologías a la difusión de sus obras de arte, se han producido reclamaciones de piezas de quienes se consideran sus legítimos propietarios. Cuando el cedente o propietario de la obra es un museo estatal, nos encontramos con el muro infranqueable de las inmunidades del Estado prestador en el territorio del foro, en su doble vertiente: de jurisdicción y de ejecución. La inmunidad de los préstamos de obras de arte es tan solo una aplicación práctica de estas inmunidades conocidas, cuyo alcance depende de la legislación interna del Estado en el que tiene lugar el procedimiento

* Licenciada y Doctora en Derecho por la Universidad Autónoma de Madrid; M. A. en Relaciones Internacionales por el Instituto Universitario de Investigación Ortega y Gasset de Madrid (Universidad Complutense de Madrid); Centre for Studies and Research de la Academia de Derecho Internacional (La Haya, Holanda). Catedrática acreditada por el Ministerio de Educación, Cultura y Deporte (Resolución del 4 de julio de 2012). En la actualidad, se desempeña como profesora titular de Derecho Internacional Público y Relaciones Internacionales en la Universidad Autónoma de Madrid. Correo electrónico: s.torrecuadrada@uam.es

Para citar este artículo: Torrecuadrada García-Lozano, S. "Las obras de arte del Estado y su inmunidad", Anuario Colombiano de Derecho Internacional (ACDI), 2017, 10, pp. 401-426. Doi: 10.12804/revistas.urosario. edu.co/acdi/a.5301 
judicial o de aquel en el que se solicita la ejecución. Los Estados, para evitarlo, suelen celebrar acuerdos bilaterales que les permiten garantizar el retorno de las colecciones que prestan. Ello evidencia el difícil equilibrio entre las inmunidades del Estado, el derecho a la propiedad individual y el derecho a la tutela judicial efectiva; o entre el interés colectivo — la cooperación cultural—y el individual — la recuperación del bien-.

Palabras clave: Estado, inmunidad de jurisdicción, inmunidad de ejecución, tribunales nacionales e internacionales, préstamos.

\section{Cultural Property of the State and its Immunity}

Abstract: In recent times, technology has had the effect of making the world smaller in many respects. Nowadays not only it is possible to travel distances in shorter time, but we also have the possibility of accessing 'satellite' museums of the Hermitage Museum of Saint Petersburg in, for example, Malaga or Amsterdam, or enjoying collections lent by museums to others that are located in the other side of the world than where they are usually exhibited. This lending and the application of new technologies for the diffusion of art works have led to reclamations of pieces by those who consider themselves legitimate owners. When the assignor or the owner is a State museum we are faced with the insurmountable wall of state immunity of the lending State in the territory of the forum state in two aspects: jurisdiction and execution. The immunity of the lent art works is merely a practical application of these known immunities whose scope depends on the internal legislation of the State in which the judicial proceedings take place or in which a claim for execution of a judgement is made. The states, to avoid it, usually conclude bilateral agreements that allow them to guarantee the return of lent collections. This practice demonstrates the difficult equilibrium between State immunity, the individual right to property and the right to effective judicial remedies, or between the collective interest — cultural cooperation — and the individual — the recuperation of the good-.

Key words: State, jurisdictional immunity of the State, measures of constraint, international courts and national courts, loans. 
Resumo: Nos últimos tempos, a tecnologias tem tido por efeito diminuir o mundo em todos os sentidos. Assim, agora não só as distâncias recorremse em menos tempo, mas para além temos ocasião de contemplar museus "satélites" do Museu Hermitage de São Petersburgo em Málaga ou Amsterdã, por exemplo, ou coleções emprestadas por museus a outros para que disfrutemos com elas no outro lado do mundo de onde habitualmente expõem-se. Com ocasião destes empréstimos e a aplicação de novas tecnologias à difusão das suas obras de arte, se têm produzido reclamações de peças de quem consideram-se os seus legítimos proprietários. Quando o cedente ou proprietário da obra é um museu estatal, encontramo-nos com o muro infranqueável das imunidades do Estado emprestador no território do fórum, na sua dupla vertente: de jurisdição e de execução. A imunidade dos empréstimos de obras de arte é tão só uma aplicação prática destas imunidades conhecidas, cujo alcance depende da legalização interna do Estado no que tem lugar o procedimento judicial ou aquele no que solicita a execução. Os Estados, para evitá-lo, costumam celebrar acordos bilaterais que lhes permitem garantir o retorno das coleções que emprestam. Isso evidencia o difícil equilíbrio entre as imunidades do Estado e o direito à propriedade individual e o direito à tutela judicial efetiva; ou entre o interesse coletivo —a cooperação cultural— e o individual —a recuperação do bem-.

Palavras-chave: Estado, imunidade de jurisdição, imunidade de execução, tribunais nacionais e internacionais, empréstimos.

\section{Introducción}

En los últimos tiempos, la tecnología ha conseguido empequeñecer el mundo, ahora podemos encontrarnos con museos 'satélites' de grandes museos separados por una distancia de casi cinco mil kilómetros (entre el Museo Hermitage de San Petersburgo y el de Málaga) o de préstamos de colecciones que viajan más de siete mil quinientos kilómetros (entre el Museo Pushkin de Moscú y el moma de Nueva York) para exponer sus obras. Estos desplazamientos y la disposición a través de internet de los catálogos de las colecciones de los museos han tenido un efecto colateral indeseado por parte de los prestatarios de aquellas obras de arte: la reclamación, de quienes se consideraban sus legítimos propietarios o de sus 
herederos, de bienes que fueron nacionalizados (sin compensación alguna) como consecuencia de la Revolución Soviética, incautados por los nazis o vendidos a bajo precio para poder huir del horror que supuso el nazismo (especialmente para el pueblo judío) desde la década de los años treinta del siglo pasado hasta finalizar la Segunda Guerra Mundial.

Frente a estas reclamaciones, los Estados y museos no han permanecido neutrales, bien al contrario, han proclamado y mantenido formalmente su compromiso (político) de facilitar el regreso de estos bienes a sus legítimos propietarios. Sin embargo, frente a las demandas interpuestas en aras a la recuperación de las obras en cuestión, han alegado sistemáticamente la inmunidad de la que son titulares (en el caso de los Estados) de conformidad con el derecho internacional para evitar la pérdida de esos valiosos objetos. En todo caso, tanto los Estados como los museos han participado (a partir de los años noventa) en la elaboración de textos de naturaleza política en los que declaraban su compromiso de adoptar cuantas medidas fueran precisas con el propósito de proceder a la devolución de aquellos bienes a los que fueron sus propietarios o a sus herederos.

No obstante, estos compromisos no pueden conducirnos a error, pues, si bien resulta innegable que han procedido a la devolución de múltiples obras de arte a los propietarios anteriores a la incautación o a sus herederos, ${ }^{1}$ en muchas más ocasiones de las deseadas los Estados han interpuesto la excepción de inmunidad de jurisdicción en un intento de evitar el conocimiento sobre el fondo de la demanda restitutoria de la obra u obras de arte y/o de que se ejecute la sentencia dictada. ${ }^{2}$

Los museos, a través del International Council of Museums (ICOM), ${ }^{3}$ adoptaron en 1999 las ICOM recommendations concerning the return of works of art belonging to jewish owners, ${ }^{4}$ en las que, entre otras, se incorpora la recomendación siguiente: "To actively address the return of all objects of art that formerly belonged to Jewish owners or any other owner, and that are now in the possession of museums, to their rightful owners or their heirs, according to national legislation and where the legitimate ownership of these objects can clearly be established".

\footnotetext{
$1 \quad V i d$. a modo de ejemplo los informes austríacos al respecto en http://www.artrestitution.at/Reports_2.html.

2 Vid. infra caso Noga en el epígrafe II.2.1.

3 Sobre esta organización no gubernamental vid. http://icom.museum/

$4 \quad$ Vid. en lootedart.com/OXSHM8852201_print

5 Id. nota anterior.
} 
Por otra parte, a diferencia de lo que ocurre con los bienes expropiados ilícitamente como consecuencia de la Revolución Soviética (que fueron nacionalizados y permanecen en museos estatales), la incautación de obras de arte por parte del nazismo plantea múltiples problemas, derivados tanto de las dimensiones de la contienda como de sus consecuencias (como, por ejemplo, la posterior redefinición de fronteras). Si añadimos a lo anterior el transcurso del tiempo, el desplazamiento tanto de los bienes objeto de reclamación como de los anteriores propietarios o sus herederos, o las sucesivas transacciones de las obras de arte reclamadas, la complejidad de las cuestiones en presencia se multiplica considerablemente. ${ }^{6}$

La cuestión por plantear ahora es: ¿el derecho internacional contiene una obligación de restituir ${ }^{7}$ el bien ilícitamente apropiado? El Protocolo Primero a la Convención para la Protección de los Bienes Culturales en Caso de Conflicto Armado, de 1954, establece la obligación de restitución de los bienes incautados durante el período de la contienda bélica ${ }^{8}$ (I.3), por lo que los Estados que sean partes en la Convención y en este

6 En cuanto a las obras incautadas por el nazismo, vid. la Declaración Conjunta Adoptada por las Potencias Aliadas el 5 de enero de 1943, que establecía el compromiso de colaborar para evitar los efectos del expolio, negando además la validez de las confiscaciones directas e indirectas —entendiendo por tales las compraventas forzosas- en los territorios ocupados. Este texto despliega un doble efecto jurídico: 1) en relación con los formulantes, obligados jurídicamente por él debido a su naturaleza convencional; 2) la declaración de nulidad de la confiscación no resulta oponible a un Estado distinto de los suscriptores, aunque posteriormente se extiende su oponibilidad al reiterarse la nulidad en los tratados de paz celebrados al finalizar la contienda, de los que sí son partes los vencidos. Sobre las distintas opiniones y posturas de quienes negociaron el texto, vid. Kurtz, M. J., "The allied struggle over cultural restitution, 1942-1947”, en International Journal of Cultural Property, 2010, (17), pp. 177-194.

7 Distinguimos entre devolución y restitución, pues esta última es una consecuencia de la responsabilidad internacional que implica (artículo 35 del proyecto de artículos de la CDI sobre responsabilidad internacional del Estado por hechos internacionalmente ilícitos) la obligación del Estado a quien resulta atribuible el ilícito de proceder a "restablecer la situación que existía antes de la comisión" de aquel, por lo tanto, el obligado a la restitución es el responsable de la privación. El elemento de responsabilidad no siempre está presente en los supuestos de hecho en presencia en este trabajo, puesto que, en múltiples ocasiones, el responsable de la privación no es el que lo posee en la actualidad. A pesar de lo cual, utilizaremos indistintamente restitución y devolución como términos sinónimos para evitar reiteraciones.

Vid. el texto en su versión oficial en http://portal.unesco.org/es/ev.php-URL_ ID $=15391 \&$ URL_DO=DO_TOPIC\&URL_SECTION $=201 . h t m l$ 
Protocolo están jurídicamente obligados a devolver los bienes culturales ajenos que se encuentren en su territorio.

Si bien la respuesta es clara, de acuerdo con lo recién indicado, cabe plantear, como se apuntaba en 1954, la existencia de un tercero adquirente de buena fe. ${ }^{9}$ Para ello el mismo Protocolo indica que procede la restitución con indemnización a este último. Será el Estado que "tuviera la obligación de impedir la exportación de bienes culturales del territorio ocupado" quien asuma los gastos de la indemnización (I.4), o, lo que es lo mismo: el incumplidor de la obligación de control asume las consecuencias de su comportamiento. Por su parte, el Convenio Unidroit de 1995 sobre bienes culturales robados o exportados ilícitamente sustituye la buena fe por la diligencia debida, más fácil de probar ateniéndose a los elementos objetivos en presencia, ${ }^{10}$ correspondiendo al tercero de buena fe igualmente una indemnización equitativa, que asumirá (de ser posible) aquel de quien adquirió esa pieza o cualquier otro cedente anterior.

En todo caso, la responsabilidad subyacente en los supuestos planteados en ambos tratados internacionales es el mismo: habrá de proceder a indemnizar aquel que cometió el hecho ilícito, que en el caso del Protocolo de 1954 será quien vulneró su obligación de impedir el desplazamiento, mientras en el texto de 1995 es el que no actuó con la debida diligencia a la hora de ofrecer en compraventa un bien, repercutiendo así la responsabilidad en quien adquirió fraudulentamente el bien.

Podemos intentar otra respuesta a la cuestión planteada en función de quien posea en la actualidad el bien reclamado: el Estado responsable de su incautación ilícita o un tercero adquirente de buena fe. Si el Estado responsable fuera el autor de la apropiación ilícita, nunca habría adquirido su propiedad debido a la nulidad de la transferencia, por lo que tiene que asumir las consecuencias de la responsabilidad internacional derivada de aquel: la restitución del bien. Las palabras de Max Huber en el arbitraje sobre la Isla de Palmas alcanzan la misma conclusión de modo muy gráfico: "It is evident that Spain could not transfer more rights than she herself possessed". ${ }^{11}$

9 Sobre el principio de buena fe en los conflictos que versan sobre el patrimonio cultural robado, vid. Symeonides, S. C., "A choice-of-law for conflicts involving stolen cultural property", en Vanderbilt Journal of Transnational Law, 2005, (38), pp. 1177-1198, en particular pp. 1190-1191.

$10 \mathrm{Vid}$. artículo $4^{\circ}$ del convenio referido en el texto en la siguiente dirección electrónica: http://www.unidroit.org/spanish/conventions/1995culturalproperty-convention-sp.pdf

11 Vid. en http://web.archive.org/web/20080528174538/http://www.gwu.edu/ jaysmith/ Island.html 
Si, por el contrario, el poseedor actual adquirió de buena fe esa obra de arte, se plantean dos cuestiones: 1) la identificación de la presencia de la buena fe o la actuación con la diligencia debida; y 2) si podría considerarse propietario al último poseedor. En relación con la primera, carecemos de una definición positiva que nos permita proceder a la necesaria identificación, pero existen elementos que nos ayudan a probar si se ha actuado con la debida diligencia. En este sentido, el artículo 4.4. del Convenio Unidroit indica la necesidad de observar todas las circunstancias en presencia, "en particular la calidad de las partes, el precio pagado, la consulta por el poseedor de cualquier registro relativo a los bienes culturales robados razonablemente accesible y cualquier otra información y documentación pertinente que hubiese podido razonablemente obtener, así como la consulta de organismos a los que podía tener acceso o cualquier otra gestión que una persona razonable hubiese realizado en las mismas circunstancias".

Idéntico criterio utiliza la Directiva de la Unión Europea relativa a la restitución de bienes culturales que hayan salido de forma ilegal del territorio de un Estado miembro, ${ }^{12}$ que aporta elementos objetivos sustantivamente similares a los recién transcritos, aunque con algunas precisiones que expresamente no se contemplan en ellos. ${ }^{13}$ En consecuencia, en la Unión Europea, solo los poseedores de bienes que cumplan las condiciones establecidas en la citada norma podrán percibir la indemnización que determine el tribunal competente del Estado requerido a efectos de la restitución del bien que sufragará el Estado miembro requirente. Obsérvese que, en el régimen general establecido por la Directiva, la posesión ilícita

12 Es la Directiva 2014/60/UE, del 15 de mayo de 2014, por la que se modifica el Reglamento UE 1024/2012, vid. en Diario Oficial de la Unión Europea, No L159, pp. 1-10. El artículo 2.3 del Código Deontológico del ICOM no se aparta de esta idea, pues indica la conveniencia de realizar todos "los esfuerzos necesarios para asegurarse de que un objeto ofrecido en compra, donación, préstamo, legado o intercambio no ha sido adquirido o exportado ilegalmente de su país de origen o de un país en tránsito en el que hubiera podido ser poseído legalmente, incluido el país en que se encuentra el museo. A este respecto, se debe obrar con la debida diligencia para reconstituir el historial completo del objeto desde su descubrimiento o creación". Vid. en http://archives.icom.museum/code2006_spa.pdf 13 El artículo 10.2 dice: "Todas las circunstancias de la adquisición, en particular la documentación sobre la procedencia del bien, las autorizaciones de salida exigidas por el derecho del Estado miembro requirente, en qué calidad actúan las partes, el precio pagado, la consulta por el poseedor de los registros accesibles sobre bienes culturales robados y cualquier otra información pertinente que hubiese podido razonablemente obtener o cualquier otra gestión que una persona razonable hubiese realizado en las mismas circunstancias". 
no se sana con la donación o la sucesión, pues "el poseedor no podrá disfrutar de un régimen más favorable que el que haya tenido la persona de quien haya adquirido el bien en dicho concepto".

El propósito de las páginas que siguen es la inmunidad del Estado respecto de las obras de arte, debido a su potencial para impedir, dificultar o, cuando menos, retrasar la recuperación de aquellas por parte de sus propietarios anteriores. Con este objetivo profundizaremos las distintas cuestiones hasta aquí indicadas. Para ello, en primer lugar, nos detendremos en las inmunidades clásicas de jurisdicción y de ejecución; y, en segundo término, en la aplicación particular que suponen ambas en los bienes en préstamo, para terminar con un apartado dedicado a conclusiones.

\section{La inmunidad de jurisdicción y ejecución del Estado extranjero}

En este epígrafe nos vamos a centrar en la inmunidad de las obras de arte, utilizando un criterio cronológico de los tipos de inmunidad más conocidos (de jurisdicción y de ejecución), comenzando por la primera materialización que se produce en el tiempo, pues solo existe la posibilidad de ejecución forzosa cuando se dota a la inmunidad de jurisdicción de un alcance relativo, que en la actualidad se aplica por la mayoría de los tribunales nacionales. ${ }^{14}$

\subsection{La inmunidad de jurisdicción}

En relación con las obras de arte, la inmunidad de jurisdicción no plantea particularidad alguna respecto de otras materias, aunque solo se aplica a los museos estatales, al tratarse de entidades públicas que "son por sí mismos órganos estatales autónomos que responden ante el gobierno central o ante uno de sus departamentos, o son administrados por él". ${ }^{15}$

\footnotetext{
14 Vid. en este sentido el Projet Pilote du Conseil de l'Europe sur la pratique des Etats relative aux immunités — Rapport Analytique, del Comite des Conseillers Juridiques sur le droit international public (CAHDI), 30ª reunión del 19-20 de septiembre. Doc. CAHDI (2005) 5 Final, p. 121, parágrafo 437-.

15 Vid. el tercer informe sobre las inmunidades jurisdiccionales de los Estados y de sus bienes, por el Sr. Sompong Sucharitkul, relator especial, documento A/CN.4/340 y add. 1, p. 152, parágrafo 37.
} 
Por otra parte, la apreciación de esta inmunidad depende del tipo de acto en el que se fundamente el procedimiento iniciado. Así, del artículo 10 de la Convención de las Naciones Unidas de 2004 sobre las Inmunidades del Estado y de sus Bienes ${ }^{16}$ (en adelante, Convención de 2004), se desprende que el fruto de una transacción mercantil ${ }^{17}$ entre museos estatales gozaría de inmunidad, por resultar una excepción prevista en este artículo. Igualmente, el texto convencional prevé que los Estados mediante tratados puedan proteger la transacción mercantil a estos efectos.

Por otra parte, si el origen de una reclamación de la obra de arte en cuestión versara sobre "un proceso relativo a la determinación de: a) un derecho o interés del Estado respecto de bienes inmuebles situados en el Estado del foro", su posesión o uso, así como al esclarecimiento de b) "un derecho o interés del Estado respecto de bienes muebles o inmuebles, nacido en virtud de sucesión, donación u ocupación vacante", no cabría la inmunidad de jurisdicción, razonablemente, puesto que el Estado actúa al margen de la categoría de actividades iure imperii.

La jurisprudencia nacional interpreta las excepciones de conformidad con su ordenamiento interno, siempre que este haya determinado el alcance preciso de estas inmunidades. El caso de Estados Unidos resulta especialmente interesante, por ser el que nos ha proporcionado más práctica de reclamaciones de obras de arte tanto expropiadas tras la Revolución Bolchevique y, posteriormente, ubicadas en la antigua Unión Soviética, como de bienes incautados por el régimen nazi. Según los pronunciamientos judiciales estadounidenses, la inmunidad hay que apreciarla en cada caso concreto teniendo en cuenta todas las circunstancias en presencia. ${ }^{18}$

El asunto de la colección Herzog (cuyo propietario poseía unas 2.500 obras de arte en el período de entreguerras) contra Hungría resulta ilustrativo en este punto, pues su supuesto de hecho responde básicamente a los parámetros de la incautación de bienes culturales producida durante la

16 A día 15 de diciembre de 2015, faltan diez instrumentos de ratificación o adhesión para su entrada en vigor (artículo 30.1). Vid. en http://treaties.un.org

17 El concepto de transacción mercantil se encuentra en el artículo 2.1.c) de la Convención de 2004. Sobre la excepción de transacción mercantil, ver el cuarto informe sobre las inmunidades jurisdiccionales del Estado y de sus bienes, en http://legal.un.org/ilc/ documentation/spanish/a_cn4_357.pdf

18 Sobre el asunto Cassirer contra el Reino de España y la Fundación Thyssen-Bornemisza, vid. el trabajo de Caamiña, C., "Inmunidad de jurisdicción y plazos de prescripción: caso Cassirer", en Anuario de la Facultad de Derecho de la UAM, 2015, 19, pp. 79-100. 
época del nazismo alemán. Las propiedades del barón Mor Lipot Herzog, ciudadano judío húngaro, fueron confiscadas por el gobierno, durante su colaboración con la Alemania nazi. Tras la Segunda Guerra Mundial, los herederos del barón intentaron sin éxito recuperar sus propiedades, que permanecían en el Museo de Bellas Artes de Budapest, iniciando algunos de ellos un procedimiento contra la República de Hungría, la Galería Nacional, el Museo de Bellas Artes, el Museo de Artes Aplicadas, así como la Universidad de Tecnología y Economía de Budapest. La defensa húngara alegó la excepción de inmunidad del Estado, de la que es titular de acuerdo con la ley estadounidense (Foreign State Immunity Act, FSIA), ${ }^{19}$ salvo que se produzcan unas circunstancias ausentes en el caso en presencia, adoptando la norma interna idéntico punto de partida que la Convención de 2004.

El Tribunal de Apelaciones del Circuito del Distrito de Columbia ${ }^{20}$ entendió que "the seizure of the Herzog Collection by Hungary alone would not constitute a violation of international law", debido a que "Hungary did not consider M. Nierenberg and Ms. Weiss de Csepel to be Hungarian citizens at the time of the seizures". ${ }^{21}$ Ciertamente, los propietarios de las obras de arte carecían de la nacionalidad húngara porque al amparo del nazismo se privó a las personas judías de su nacionalidad en los territorios por ellos ocupados o cuyos gobiernos colaboraban con aquel régimen. La familia Herzog invocó entonces la actividad comercial del Estado demandado, que, de acuerdo con el mismo ordenamiento estadounidense, impide la consideración de la inmunidad. Para poder tener en cuenta esta excepción, es preciso reunir una serie de requisitos ${ }^{22}$ aparentemente presentes en el caso. Así, los hechos que provocaron la interposición de la demanda se produjeron en

19 Vid. 28 U.S. Code Chapter 97 - Jurisdictional immunities of foreign States, en http:// www.law.cornell.edu/uscode/text/28/part-IV/chapter-97, al que precedió en el tiempo el Immunity from Seizure Act (IFSA), aprobado por el Congreso el 19 de octubre de 1965, 22 U.S.C. \$2459, vid. en http://www.state.gov/s/1/3198.htm

20 Sentencia del 19 de abril de 2013, usCa Caso 11-7096, David L. de Csepel y otros contra la República de Hungría, Estado extranjero y otros.

21 Id. sentencia citada en la nota anterior, p. 9.

22 Tales requisitos son: "1) the lawsuit must be based upon an act that took place outside the territory of the United States; 2) the act must have been taken in connection with a commercial activity [;] and 3) the act must have caused a direct effect in the United States'... Because Hungary's actions obviously occurred outside the United States, the exception's applicability turns on 1) whether Hungary's repudiation of bailment agreements with respect to the Collection constitutes an act taken in connection with a commercial activity and 2) whether this act caused an 'effect direct' in the United States". Id. nota 20, p. 11. 
territorio húngaro; se entiende que la negativa de Hungría a cumplir los contratos de comodato que había celebrado con los herederos del barón Herzog $^{23}$ son un elemento que demuestra el cumplimiento de la conexión con actividades comerciales y que provoca un efecto directo en Estados Unidos, cumpliéndose también esta condición.

En consecuencia, es la legislación interna del Estado del foro la que va a determinar tanto el alcance de la inmunidad (siempre respetando los límites establecidos por el derecho internacional) como de sus excepciones y las condiciones de aplicación a cada caso con el propósito de analizar la competencia de sus tribunales.

\subsection{La inmunidad de ejecución}

Acerca de la inmunidad frente a la ejecución forzosa de las sentencias dictadas, la Convención de 2004 (artículo 19) establece que solo podrá procederse "contra bienes de un Estado, en relación con un proceso ante un tribunal de otro Estado" si media consentimiento del afectado, si existen bienes afectos a fines distintos de los oficiales no comerciales o si se ha indicado el bien en cuestión para este propósito.

De lo anterior se desprenden dos impedimentos en relación con las obras de arte estatales: 1) las obras de arte no suelen utilizarse para fines comerciales, ${ }^{24}$ puesto que se trata de piezas expuestas; y 2) pueden carecer del nexo requerido con la entidad contra la que se ha iniciado el proceso (exigido por el artículo 19 de la Convención de 2004). En la mayoría de las ocasiones se evitaría el último impedimento, pues normalmente, cuando se insta la ejecución forzosa de una sentencia sobre una obra de arte, se exige la restitución precisamente de ese bien, aunque el poseedor actual no siempre coincide con el causante de la privación.

Por otra parte, el artículo 21 del mismo texto concreta las clases especiales de bienes que, estando ubicados en un Estado extranjero, gozan de protección especial, impidiendo su ejecución forzosa; entre ellas se encuentran “d) los bienes que formen parte del patrimonio cultural del

23 En virtud de los cuales el Estado retenía la posesión, comprometiéndose a devolverlos a los propietarios, y que Hungría había incumplido. Vid. la sentencia citada supra en nota 20 , pp. 2 y ss.

24 En el Reino Unido, el State Immunity Act de 1978 parte de la prohibición de ejecutar forzosamente medidas contra los Estados extranjeros salvo "in respect of property which is for the time being in use or intended for use for comercial purposes”, art. 13(4). 
Estado, o parte de sus archivos, y no se hayan puesto ni estén destinados a ser puestos en venta; e) los bienes que formen parte de una exposición de objetos de interés científico, cultural o histórico y no se hayan puesto ni estén destinados a ser puestos en venta".

Por lo demás, los bienes que formen parte del patrimonio cultural de un Estado en el extranjero se encuentran protegidos con independencia del lugar en el que se ubiquen. Lo habitual, de no exhibirse en una exposición, es que decoren una Embajada, gozando de una doble protección: la derivada de la naturaleza de los bienes y otra de la inviolabilidad de la que gozan las misiones diplomáticas según la Convención de Viena sobre relaciones diplomáticas (artículo 22).

Del mismo modo que en la inmunidad de jurisdicción, por lo que se refiere a la de ejecución, los ordenamientos internos protagonizan la determinación del alcance de las normas internacionales, permitiendo su aplicación a los casos concretos que ha de resolver el órgano interno encargado de su solución. Igual que en aquel caso, entre los ordenamientos nacionales encontramos distintos modelos, como los que rechazan la inmunidad de ejecución, lo que aparentemente (según August Reinisch) ocurre en Turquía. ${ }^{25}$ En otros casos, como el de Croacia o Grecia, la ejecución forzosa requiere de una autorización del ejecutivo, ${ }^{26}$ lo que no proporciona un método que aporte un criterio objetivo para que nos permita realizar un análisis predictivo, pues la decisión parece depender, en mayor o menor medida, de criterios de oportunidad política. ${ }^{27}$

Por su parte, el Tribunal Federal suizo acogió en 1986 la inmunidad de ejecución sobre el local en el que estaba ubicada la Casa de España en Zúrich, atendiendo a su función cultural. ${ }^{28} \mathrm{El}$ supuesto encontraba su ori-

25 Vid. Reinisch, A., "European Court practice concerning State immunity from enforcement measures", en The European Journal of International Law, 2006, 17, pp. 803-816; la cita del texto se encuentra en la p. 813.

26 Vid. el artículo 18 del Croatian Execution Act traducido en http://www.vsrh.hr/ CustomPages/Static/HRV/Files/Legislation_Execution-Act.pdf

27 Vid. el documento preparado por el Committee of Legal Advisers on Public International Law (CAHDI), en su $24^{a}$ reunión celebrada en Bratislava, entre el 9 y 10 de septiembre de 2002 "Pilot Project of the Council of Europe on State Practice Regarding State Immunities/Projet Pilote du Conseil de l'Europe sur la pratique des Etats relative aux immunites", en http://www.coe.int/t/dlapil/cahdi/Source/Docs2002/CAHDI_2002_12bil.pdf

28 Vid. sentencia del 30 de abril de 1986, en el caso Royaume d'Espagne c. Entreprise X, Office de poursuites de Berne et Président du Tribunal de Berne IV, comentario firmado por Lucius Caflisch, en Annuaire Suisse de Droit International, 1987, XLIII, pp. 158-162. 
gen en la cancelación de una "adjudicación directa de las obras de reforma y ampliación” de la Casa de España, ${ }^{29}$ por lo que la sociedad adjudicataria solicitó el embargo de los inmuebles pertenecientes al Estado español y en los que se ubicaba la sede de la citada Casa. El Tribunal se cuestionó sobre la finalidad a la que se destinaba el inmueble con el propósito de averiguar si podía ser objeto de la medida solicitada por el demandante. Cuando España adquirió uno de aquellos locales, alegó su intención de ubicar en él un centro educativo, cultural y de esparcimiento de los trabajadores españoles en Suiza, para impedir la quiebra de los vínculos existentes con España. Extinguido el centro, en su lugar se instaló la Casa de España con la misma finalidad, por lo que no se encontraba en sentido estricto afecto a actividades soberanas del Estado, pero tampoco resultaba afín a un ejercicio iure gestionis, por lo cual el Tribunal entendió la presencia en el supuesto de la inmunidad de ejecución del Estado español.

Años después, la Corte Internacional de Justicia (CIJ, en adelante) se encontró con un supuesto que recuerda al recién referido, centrado en la inmunidad de un bien inmueble en el que se aloja la asociación ítaloalemana para el acercamiento científico-cultural entre los dos países: se trata del asunto que enfrentó a Alemania e Italia con intervención de Grecia. ${ }^{30} \mathrm{La}$ CIJ resolvió negativamente una demanda interpuesta como consecuencia de la ausencia de indemnización a los trabajadores forzosos griegos e italianos durante la época de la Alemania nazi. Las víctimas de esos hechos habían acudido a la jurisdicción italiana que acogió sus demandas considerando que la inmunidad del Estado no se aplicaba cuando el acto origen de la diferencia es un crimen internacional, lo que respondía al supuesto fáctico en presencia. ${ }^{31}$

Alemania respondió a esta medida demandando a Italia por vulnerar la inmunidad de jurisdicción de la que es titular conforme con el derecho internacional público. El edificio en cuestión se destina a actividades de

\footnotetext{
29 Vid. la orden del 7 de enero de 1986, por la que se publica la declaración de nulidad del contrato de ejecución de obras en la Casa de España en Zúrich, en BOE $\mathrm{N}^{\circ}$ 37, del 12 de febrero de 1986, pp. 5709-5710.

30 Vid. la sentencia del 3 de febrero de 2012 en el asunto de las Inmunidades jurisdiccionales del Estado (Alemania c. Italia); Grecia (interviniente), en http:/ /www.icj-cij.org/ docket/files/143/16884.pdf

31 Vid. parágrafo 109 de la sentencia citada en la nota anterior, pp. 145-146.
} 
carácter cultural (igual que en el supuesto de la Casa de España), cuya protección deriva de encontrarse afecto a aquel propósito. ${ }^{32}$

En consecuencia, la jurisprudencia nacional e internacional afirma que la inmunidad de ejecución alcanza a bienes afectos a actividades culturales, aunque los edificios a los que se protege de este modo no se encuentran en ninguna de las categorías establecidas en los tratados internacionales que contienen un concepto de tales bienes. Enseñándonos además, en el caso de los tribunales nacionales especialmente, la repercusión política de las decisiones adoptadas, puesto que, en ocasiones, supuestos aparentemente similares se resuelven de modo diferente, atendiendo a la presión que es capaz de soportar el Estado afectado y a la calidad, también, por qué negarlo, del sujeto que presiona en cada caso.

\section{La inmunidad de los bienes artísticos en préstamo}

El desplazamiento de obras de arte es consecuencia de préstamos entre instituciones estatales o coleccionistas privados que permiten materializar la idea de la cooperación cultural internacional, que es uno de los propósitos de las Naciones Unidas. ${ }^{33}$ Los préstamos de obras de arte resultan muy enriquecedores desde una perspectiva cultural, al promover la tolerancia, un valor deseable en todas las sociedades, además, como afirma Norman Palmer, "it has long been accepted that art loans are a cardinal form of modern cultural exchange. To some they are also a notable element in the 'soft power' exerted by civilized states". ${ }^{34}$

En este epígrafe responderemos a la cuestión sobre si las inmunidades del Estado alcanzan a los supuestos en los que la obra de arte en cuestión ha sido prestada, por ejemplo, para su exhibición en un museo de un tercer Estado donde se encuentra cuando se solicita su embargo

\footnotetext{
32 Estaría al margen de lo establecido tanto en el artículo $1^{\circ}$ de la Convención de 1954, que, si bien alcanza bienes inmuebles, no a este que no encaja dentro de las categorías así establecidas en el citado precepto, como de la Convención de 1970 y la del Unidroit de 1995, ambas destinadas a evitar la importación y exportación de bienes culturales, sin contemplar los bienes inmuebles.

33 La Carta de las Naciones Unidas se refiere a la cooperación cultural en distintos preceptos, entre ellos en los artículos 1.3 y 13.1.

34 Vid. Palmer, N., "Adrift on a sea troubles: crossborder art loans and the specter of ulterior title", en Vanderbilt Journal of Transnational Law, 2005, 38, (4), pp. 947-996; la transcripción del texto es de la p. 949.
} 
a los tribunales. Para lograr nuestro propósito, estructuraremos este epígrafe en dos partes: 1) para intentar resolver las cuestiones previas cuyo esclarecimiento nos permitirá comprender mejor las dos siguientes; y 2) el análisis de la jurisprudencia.

\subsection{Cuestiones previas}

Para responder la pregunta que vertebra este epígrafe, hemos de calificar los préstamos como acto iure imperii o acto iuri gestionis, para determinar si nos encontramos ante el presupuesto necesario de la inmunidad. Si lo consideramos iure imperii, la excepción procesal prosperará, pero, si resulta ser iure gestionis, los tribunales habrán de comportarse como lo haría si el demandado fuera un particular.

En el supuesto de los préstamos de obras de arte, tanto los Estados como los museos estatales, privados o coleccionistas pueden actuar como prestadores y prestatarios, lo que nos ubica en la segunda categoría indicada. A pesar de lo cual, M. Weller establece "the assumption of immunity for artworks on loan from foreign states, however is based on the condition that the purpose of the loan is one de ius imperii", 35 afirmando su naturaleza consuetudinaria cuando el propietario de la obra prestada es el Estado. ${ }^{36}$ Ello aunque el comportamiento tiene claros tintes comerciales, al tratarse de préstamos compensados, financiados por el museo prestatario mediante la venta de entradas o de productos de la exposición, ${ }^{37}$ por lo que encajaría mejor como acto de comercio, por ende, iure gestionis, en el que no cabe aplicar la inmunidad.

Claro que, estando de acuerdo con lo anterior, la práctica apunta en sentido contrario al aquí reflejado, pues las obras de arte son elementos relevantes de la identidad nacional, ${ }^{38}$ lo que nos conduce a preguntarnos si

35 Weller, M., "Immunity for artworks on loan? A review of international customary law and municipal anti-seizure statutes in light of the Liechtenstein litigation", en Vanderbilt Journal of Transnational Law, 2005, (38), pp. 997-1039; el texto transcrito se encuentra en la p. 1009.

36 Id. nota anterior, pp. 1014-1015.

37 Ver en este sentido Pop, L., "Arrestuing art loan seizures", en Columbia-ILA Journal of Law and Arts, 2001, 24, (2), pp. 213-233; la idea del texto se encuentra en la p. 231.

38 Jayme, E., "Globalization in art law", en Vanderbilt Journal of Transnational Law, 2005, 38, pp. 927-945, especialmente pp. 933 y ss. 
todas las obras de arte que figuran en los museos estatales conforman esta categoría de identidad estatal; así, por ejemplo, ¿los cuadros de Matisse que fueron adquiridos por un coleccionista privado y expropiados ilícitamente en la Revolución Bolchevique reflejan la identidad rusa? Este argumento nos llevaría a analizar la nacionalidad de las obras de arte, aspecto acerca del cual no existe acuerdo y nos aboca a debates sobre el nacionalismo o el internacionalismo cultural, alejándonos de las inmunidades. ${ }^{39}$

Regresando a la inmunidad, Nout van Woudenberg afirma que "the fact that cultural objects are immune from seizure does not automatically imply that it would be impossible to initiate legal proceedings in which the objects in question play a leading role. In general, that all depends on the laws of the forum State". 40

En la actualidad, en este ámbito material y hasta la entrada en vigor de la Convención de 2004, las normas aplicables son de naturaleza consuetudinaria, cuyos efectos (del mismo modo que las de la Convención de 2004) precisan, debido a su naturaleza non-self-executing, de normas de desarrollo adoptadas por el Estado para su aplicación. Esto supone que la viabilidad o el éxito de la reclamación interpuesta depende del derecho interno del Estado del foro, que establecerá procedimientos para lograr el objetivo marcado en la norma. En el caso de los Estados miembros de la Unión Europea, han de adaptarse además a las normas de esta organización. ${ }^{41}$ Así, en relación con la legislación alemana en este punto, Weller afirma que "the safeguarding of cultural objects on loan in Germany grounds on an administrative decision, the 'legally binding return guarantee' that bars the access of any third party claimants to the courts raising claims that conflict with the lender's claim for the recovery of the loan". ${ }^{42}$

39 Vid. Merryman, J., "Two ways of thinking about cultural property", en American Journal of International Law, 1986, 80, pp. 831-853. En la doctrina española, Pérez-Prat Durbán, L., "Las vicisitudes del patrimonio cultural: arte y derecho", en Curso de derecho internacional y relaciones internacionales de Vitoria-Gasteiz de 2006, Vitoria, pp. 241-273, vid. especialmente las pp. 247 y ss.

40 Van Woudenberg, N., "Immunity from seizure: which way leads to Rome?", actas de la conferencia dictada en Opening Session Collection Mobility 2.0., Museo Nacional del Prado, $31^{\text {st }}$ May- $1^{\text {st }}$ June 2010, Madrid, vid. en http://www.lending-for-europe.eu/filead$\mathrm{min} / \mathrm{CM} /$ public/training/madrid/van_Woudenberg-_Inmunity_from_seizure.pdf

41 Vid. Rodríguez Pineau, E. \& Martínez Capdevila, C., "La protección de los bienes culturales en la UE: un régimen puesto a prueba", en Pérez-Prat Durbán, L. \& Lazari, A. (coords.), El tráfico de bienes culturales, Tirant lo Blanch, Valencia, 2015, pp. 227-269.

42 Id. nota 35 , p. 7. 
De acuerdo con lo anterior, los tribunales nacionales se han limitado a aplicar sus respectivas legislaciones internas, sin plantearse si el comportamiento de base de la reclamación era un acto iure imperii o iure gestionis.

Los supuestos de inmunidad de ejecución de los bienes en préstamo podemos clasificarlos en dos: 1) quien desea la ejecución forzosa de una sentencia contra un Estado sobre cualquier bien que este tenga en el territorio del foro; y 2) protagonizado por la víctima de una privación ilícita de sus bienes que desea recuperar. Se trata de una clasificación conceptual, dado que las soluciones en ambos casos apuntan en una misma dirección.

El caso Noga S.A. ilustra la primera de las hipótesis indicadas, pues la sociedad pretendía la ejecución forzosa de un laudo arbitral obtenido en Suecia sobre obras de arte prestadas por el Museo Pushkin de bellas artes de Moscú para su exhibición en la Pierre Gianadda Foundation en la ciudad de Martigny ${ }^{43}$ para saldar una deuda de diez millones de dólares. Si bien la situación parecía inicialmente propicia para que Noga S.A. lograra que Rusia saldara su deuda, las presiones diplomáticas sobre el gobierno suizo motivaron el levantamiento de la orden de embargo inicial. ${ }^{44}$

Encontramos múltiples ejemplos de la hipótesis planteada en segundo lugar en distintos lugares del mundo. Así, el relativo a la colección de Shchukin, nacionalizada sin contraprestación en aplicación del Decreto del 29 de octubre de 1918. En la década de los años cincuenta, el Museo Hermitage prestó algunas de sus obras a la Maison de la Pensée Française, demandando la hija de Shchukin al gobierno soviético ante los tribunales franceses por la posesión ilícita de obras que pertenecían a su padre. ${ }^{45}$ Sus demandas han sido sucesivamente desestimadas, aunque en 1993 logró un embargo cautelar de la colección entonces prestada al Centro Georges

43 Sobre el caso vid. O'Connell, A., "The United Kingdom's immunity from seizure legislation", en Law, society, economy, working paper, en http://eprints.lse.ac.uk/24583/1/ WPS2008-20_OConnell.pdf

44 Ver en este sentido Getz, D., "Canadian immunity from seizure legislation history", en International Journal of Cultural Property, 2011, 18, pp. 201-224, la idea del texto se encuentra en la p. 206.

45 Vid. Graham, J., 'From Russia' without love: can the Shchukin heirs recover their ancestor's art collection?", en Sports and Entertainment Law Journal, 2009, pp. 66-107, en http://www.law.du.edu/documents/sports-and-entertainment-law-journal/issues/06/ From-Russia-Without-Love.pdf 
Pompidou. ${ }^{46}$ Curiosamente, en 1994 se incorpora la inembargabilidad de estos bienes en la legislación francesa. ${ }^{47}$

En el caso de Estados o museos estatales prestadores, si la legislación interna del prestatario no resulta lo suficientemente protectora de las obras de arte en préstamo, ${ }^{48}$ "... will refuse to loan without specific protection". ${ }^{49}$ Para evitarlo se observan dos posibles reacciones: 1) los Estados prestador y prestatarios celebran tratados bilaterales con el propósito exclusivo de dotar de inmunidad a las obras que van a cederse en préstamo; ${ }^{50}$ 2) los Estados prestatarios modifican su legislación con el propósito de permitir la cesión en préstamo de la colección deseada. ${ }^{51}$

Desde una perspectiva internacional, la celebración de los acuerdos bilaterales puede llevarnos a vulnerar con su contenido otras obligaciones que hayamos adquirido, pues la obligación de restitución es una consecuencia de la responsabilidad internacional derivada de la adquisición ilícita y,

46 Vid. la sentencia del 16 de junio de 1993, del Tribunal de Grande Instance de Paris, R.P. 61 655; RG 6218/93, entre Irina Shchukin (de Keller) c. le Centre National d'Art et de Culture Georges Pompidou, le Musée Hermitage (St. Petersburg) et le Musée Pushkin (Moscow).

47 La Ley 94-679, del 8 de agosto de 1994, portant diverses dispositions d'ordre économique et financier, establece en su artículo 61: «Les biens culturels prêtés par une puissance étrangère, une collectivité publique ou une institution culturelle étrangères, destinées à être exposés au public en France, son insaisissables pour la période de leur prêt à l'Etat français ou à toute personne morale désignée par lui».

48 Es el caso que relata Getz, D., en su trabajo "Canadian immunity"..., op. cit. supra nota 44, sobre la exposición "Master Paintings from the Hermitage and the State Russian Museum" en la Winnipeg Art Gallery, que fue posible gracias a la adopción del Foreign Cultural Objects Immunity from Seizure Act pocas semanas antes de que la exposición abriera sus puertas al público.

49 Vid. Weller, M., "The safeguarding of foreign cultural objects on loan in Germany", en Rivista di arte e diritto on line, 2009, (2), p. 1.

50 Vid. los tratados internacionales celebrados por Estados Unidos para proteger las colecciones que iban a recibir en préstamo por los Estados contratantes en 19 CFR $12.104 \mathrm{~g}$ - Specific items or categories designated by agreements or emergency actions (https:// www.law.cornell.edu/cfr/text/19/12.104g).

51 Podemos ilustrar esta afirmación en la exhibición de los maestros franceses rusos en la Real Academia Británica, que pudo finalmente celebrarse porque 17 días antes de abrirse la exposición en cuestión se modificó el State Immunity Act de 1978 con el objeto de introducir una parte sexta bajo el título "The tribunals, courts and enforcement act", en 2007. Vid. en Kaye, L. M., "Art loans and immunity from seizure in the United States and the United Kingdom", en International Journal of Cultural Property, 2010, 17, pp. 335-359. 
en cuanto tal, una norma consuetudinaria. En el supuesto de los bienes incautados en tiempo de guerra, se comienza a positivar con ocasión de la Conferencia de Paz de La Haya de 1907; además los Estados parte en la Convención de la Unesco de 1970 o en la del Unidroit de 1995 están obligados a restituir los bienes ilícitamente adquiridos, salvándose la contradicción entre las obligaciones derivadas de los instrumentos en presencia aplicando los criterios tradicionales al efecto: lex specialis y lexposterior.

\subsection{La jurisprudencia}

La jurisprudencia internacional se ha enfrentado en dos ocasiones a un supuesto de reivindicación de obras de arte en préstamo, ${ }^{52}$ pero, dado que en ninguna de ellas se menciona la inmunidad, no nos detendremos en ella. Sin embargo, debido a que las reivindicaciones judiciales en las que se ha planteado la inmunidad se han suscitado ante tribunales nacionales, dedicaremos este epígrafe a su análisis, que nos indica que la respuesta a la garantía de los préstamos de obras de arte depende de la legislación interna aplicable a cada caso concreto. ${ }^{53}$ Las legislaciones de Francia, Suiza ${ }^{54}$ o Alemania ${ }^{55}$ incorporan la inembargabilidad de las obras de arte en préstamo en su territorio; Bélgica, ${ }^{56}$ partiendo de esta misma premisa, excluye de la protección los bienes afectos a actividades comerciales, alcanzando la inembargabilidad a las colecciones que no "se hayan puesto ni estén

52 Vid. la sentencia del 12 de julio de 2001, del Tribunal Europeo de Derechos Humanos, en relación con la demanda del Príncipe de Liechtenstein - Hans-Adam II- contra Alemania y la sentencia del 10 de febrero de 2005 de la Corte Internacional de Justicia en el asunto relativo a ciertos bienes (Liechtenstein c. Alemania).

53 Sobre las diferentes legislaciones estatales en la materia, puede verse el estudio realizado por Knerly, S. J. Jr., Gest, K. L., Loeser, H. \& Parks, L. L. P., "International loans State immunity and anti-seizure laws", en ALI-ABA Course of study - Legal issues in museum administration, 2009, Boston, Massachusetts, en http:/ / www.lending-for-europe.eu/ filead$\mathrm{min} / \mathrm{CM} /$ public/training/Antwerp/ALI-ABA_2009_Summary_of_Seizure_Laws.pdf

54 Vid. la Ley Federal sur le transfert international des biens culturels de Suiza, del 10 de junio de 2003, en http:/ /www.wipo.int/wipolex/es/details.jsp?id=11823

55 Vid. una explicación de la ley alemana en http://www.kulturgutschutz-deutschland. de/SharedDocs/Downloads/EN/EU_Leitfaden_PDF.pdf?_blob=publicationFile

56 Vid. la ley belga que modifica el Code Judiciaire en vue d'instituer une immunité d'exécution à l'égard des biens culturels étrangers exposés publiquement en Belgique, del 14 de junio de 2004, en http://www.iec-iab.be/fr/membres/publication/annonces/ Documents/AR-du-23-juin-2004_1314.pdf 
destinadas a ser puestas en venta", como establece la Convención de 2004. Si bien la norma francesa o la belga proclaman directamente la protección de las obras de arte en préstamo, en los supuestos de Suiza y Alemania, se reconoce la protección solo en relación con los bienes respecto de los cuales la autoridad competente haya reconocido este beneficio, siendo esta declaración constitutiva de la inmunidad.

Una de las legislaciones más utilizadas ha sido la estadounidense (FSIA), que parte de la inmunidad de jurisdicción y establece la excepción de la expropriation clause, en los siguientes casos: "For any case: 1) 'in which rights in property taken in violation of international law are in issue'; 2) 'that property or any property exchanged for such property is owned or operated by an agency or instrumentality of the foreign state'; and 3) 'that agency or instrumentality is engaged in commercial activity in the United States'. 28 U.S.C. $\int 1605(a)(3)$ ". ${ }^{57}$

Se entiende por adquisición en violación del derecho internacional la producida "without payment of the prompt adequate and effective compensation required by international law' or is 'arbitrary or discriminatory in nature". ${ }^{58}$ Esto aplicado a las obras de arte supone que nunca fueron de titularidad estatal al existir un vicio en la adquisición, lo que es aplicable tanto a las nacionalizaciones bolcheviques como a las consecuencias de la aplicación de la legislación vigente en el nazismo.

Por otra parte, los tribunales estadounidenses no han exigido que la entidad que presta el bien realice una actividad comercial con identidad de bienes a los que son objeto del litigio. En uno de los casos más conocidos (la demanda de Maria Altmann contra la galería austríaca que poseía los cuadros de Klimt reclamados por la actora), fue suficiente demostrar que la demandada exponía y vendía libros en territorio estadounidense para considerar cumplido el requisito indicado. ${ }^{59}$

Sin embargo, la misma legislación permitió el regreso de los trabajos del artista ruso Kazimir Malévich, prestados por el Museo Stedelijk de Ámsterdam, cuando los herederos del pintor demandaron ante los tribunales estadounidenses al museo prestador, que los había adquirido casi cincuenta

\footnotetext{
57 La referencia al FSIA se encuentra supra en nota 19.

58 Figura a continuación del texto transcrito en el párrafo anterior, con la siguiente referencia "H.R. Rep. 94-1487, reprinted in 1976 U.S.C.C.A.N. 6604, 6618".

59 Ver la sentencia del Tribunal de Apelaciones del Noveno Circuito, del 12 de diciembre de 2002, 317 F.3d 954, Maria V. Altmann v. Republic of Austria, a Foreign State; and the Austrian Gallery, an Agency of the Republic of Austria, en http://openjurist.org/317/ f3d/954/altmann-v-republic-of-austria
} 
años antes. ${ }^{60}$ La ley estadounidense establece que, si el Departamento de Estado certifica que las obras de arte prestadas a museos estadounidenses poseen importancia cultural y que la exposición de la colección es de interés nacional, gozan de inmunidad frente a cualquier embargo judicial. ${ }^{61}$ La aplicación de esta disposición produjo una solución adversa a las pretensiones de los demandantes, al concluir que "\$2459 granting immunity and $\$ 1605$ (a) (3) establishing jurisdiction for certain claims against a foreign sovereign are both clear and not inconsistent".

El origen del caso del retrato de Wally de Egon Schiele es semejante a otros ocurridos durante la ocupación alemana de Austria, cuando Lea Bondi Jaray vendió su galería (fruto de unas técnicas cuya validez es discutible), ${ }^{62}$ en la que se encontraba el retrato de Wally que apareció en el Museo Leopold de Viena. ${ }^{63}$ Concluida la Segunda Guerra Mundial, Lea Bondi inicia los trámites ante la comisión austríaca de restitución para recuperar su colección de arte. Desde octubre de 1997 se expusieron en Nueva York cedidos en préstamo dos cuadros de Schiele, uno de ellos (Dead City III) reclamado por los herederos de un fallecido en Dachau en 1941, en relación con el cual la Fundación Leopold defendía la válida adquisición por compraventa a la cuñada del fallecido, mientras los herederos de aquel alegaban que en el supuesto en el que hubiera ocurrido la compraventa sería nula por el momento y las condiciones en las que se produjo, en aplicación de los tratados de paz de la Segunda Guerra Mundial. ${ }^{64}$ La segunda pintura prestada es el retrato de Wally, en relación con cuya adquisición la Fundación en la que habitualmente se exponía aportó una versión que contradecía tanto la de Lea Bondi como la publicada por la misma Fundación en un momento anterior.

Aunque los herederos del propietario del primer cuadro y los de Lea Bondi no habían formalizado ante los tribunales su reclamación, se adoptaron medidas para evitar la salida de las obras reclamadas del país,

60 Sentencia del 30 de marzo de 2005, del Tribunal de Distrito D. Columbia, en el asunto Malewicz v. city of Amsterdam, 362 F. Supp. 2d 298 (D.D.C. 2005).

61 Vid. 22 U.S. Code $\$ 2459$ - Immunity from seizure under judicial process of cultural objects imported for temporary exhibition or display, en https://www.law.cornell.edu/ uscode/text/22/2459

62 Vid. la sentencia del 14 de octubre de 2009, del Tribunal del Distrito Sur de Nueva York, pp. 4 y ss.

63 Vid. en http://www.leopoldmuseum.org/en/leopoldcollection/focus/Schiele

64 Vid. supra en nota 6. 
ante las protestas diplomáticas de Austria. Esta retención supuso la cancelación de préstamos internacionales de colecciones de arte. ${ }^{65}$ En 2009, el Tribunal de Distrito de Nueva York concluía que el prestador conocía la procedencia ilícita de la obra cuando la prestó al Museo de Nueva York, indicando que "the parties shall confer and inform the Court by letter no later than October 14, 2009 how they propose to proceed". ${ }^{66}$ Finalmente, el 19 de julio de 2010 (tras trece años) el conflicto se resolvió mediante un acuerdo tripartito entre Estados Unidos, el demandante y el demandado, unas semanas antes de la apertura del procedimiento civil, ${ }^{67}$ que permitió el fin de la inmovilización de las obras.

\section{Conclusiones}

Los problemas derivados de la inmunidad del Estado respecto de las obras de arte, incluso las que se encuentran en préstamo, ilustran el difícil equilibrio existente entre las inmunidades del Estado y los derechos humanos, o del interés general, que ejemplifica el Estado, y el particular, protagonizado por los propietarios o los herederos de quienes fueron privados ilícitamente de una obra de arte.

La práctica nos muestra que, a pesar de que el Estado conserve la obra de arte reclamada, los Estados tienden a evitar las dudas sobre su propiedad, debido no solo a la eventual retención cautelar de la que puede ser objeto, sino también porque con estas demandas se cuestiona la licitud de su adquisición.

Se ha advertido el protagonismo del ordenamiento interno para resolver los casos en presencia. Ello supone que, en los Estados de estructura compleja, como Canadá o Estados Unidos, la respuesta varía en función del lugar en el que se inicie el procedimiento, al gozar de la competencia legislativa en la materia los entes territoriales (Estados) y no la Unión. Ello podría provocar tantas soluciones como Estados han adoptado sus propias normas internas en la materia.

\footnotetext{
65 Vid. Getz, op. cit. nota supra 44, p. 208.

66 Vid. sentencia del Tribunal de Distrito en Nueva York en el caso United States v. Wally, 663 F. Supp. 2d 232 (S.D.N.Y. 2009), del 30 de septiembre de 2009, en https://www. courtlistener.com/opinion/2426514/united-states-v-wally/

67 http://portraitofwally.com/sites/all/articles/stipulation_and_order_of_settlement. pdf
} 
La capacidad legislativa estatal en este punto ilustra la íntima relación entre la política y el derecho, pues los criterios de oportunidad política determinan la solución aportada por el derecho en estos temas tan sensibles para los Estados, pues, si nuestro interés se focaliza en ser prestatarios de obra de arte, blindaremos las colecciones que nos ceden con medidas legislativas o tratados internacionales específicos. Si no tenemos especial interés en ello, podemos establecer cauces que permitan la recuperación de las obras de arte por sus legítimos propietarios o la ejecución forzosa de deudas de Estados extranjeros. Quizás, y dado que en el equilibrio está la virtud, debamos intentar alcanzarlo estableciendo líneas rojas que identifiquen aquellas injusticias ante las que no podemos cerrar los ojos en aras a la protección (aunque sea mínima) de los derechos humanos. Lo contrario puede conducirnos a la doble victimización de quienes fueron privados ilícitamente de sus propiedades, vulnerando con ello aquel ámbito normativo del que no podemos olvidarnos (la primera fue la pérdida ilícita del bien, la inmunidad tiene por efecto consolidar definitivamente su privación).

En todo caso, la capacidad del Estado de legislar se encuentra limitada por las obligaciones internacionales adquiridas. Más allá de las normas consuetudinarias nos espera el relativismo característico del derecho internacional, pues deberemos observar si el Estado del foro es parte en los textos de la Unesco de 1970 y/o del Unidroit de 1995. Además, los Estados miembros de la Unión Europea han de observar su ordenamiento; en relación con los Estados candidatos a la adhesión a esta organización internacional, una legislación inexistente o deficiente en este punto ha retrasado la adquisición del estatuto de miembro, como evidenció el dictamen de la Comisión Europea sobre la solicitud de adhesión a la Unión Europea de Croacia. ${ }^{68}$

El efecto que produce la inmunidad de las obras de arte de las que son titulares el Estado y los museos estatales es la discriminación, pues ante los mismos hechos objetivos se responderá de un modo u otro exclusivamente atendiendo al sujeto poseedor del bien reclamado. Si el objeto que nos fue arrebatado lo presta un coleccionista privado extranjero, no habrá problemas con la inmunidad, mientras que, si es un Estado o un museo estatal, las dificultades se multiplican por el blindaje que supone

68 Es la Comunicación COM(2014) 257 final, del 20 de abril de 2004, en http:/ / ec.europa. $\mathrm{eu} /$ transparency/regdoc/rep/1/2004/ES/1-2004-257-ES-F1-1.pdf 
la inmunidad. Por ello, la regulación nacional en desarrollo de la Convención de 2004 o de la norma general existente debería introducir como salvedad aquellos supuestos en los que el origen de la posesión estatal sea internacionalmente ilícita. Así conseguiríamos establecer un único para las inmunidades basado en la diferencia entre los comportamientos iure imperii e iure gestionis, equilibrando además la relación entre la inmunidad del Estado y los derechos humanos, en el que siempre pierde la parte más débil y, en cuanto tal, la más necesitada de protección: los derechos humanos.

La solución a estos conflictos se encuentra en la adopción de medidas preventivas con el propósito de evitar que las situaciones en presencia se repitan, como las que (con carácter recomendatorio) indica el Código Deontológico del ICOM, que establece que "un museo no debe adquirir ningún objeto o espécimen por compra, donación, préstamo, legado o intercambio sin que esté seguro de la existencia de un título de propiedad válido. Una prueba de propiedad o la posesión legal de un objeto en un país determinado no constituyen forzosamente un título de propiedad válido". En todo caso, el comportamiento adecuado a lo establecido en este código les facilitará obtener una indemnización si tuviera que devolver a sus legítimos propietarios un bien del que fueron ilícitamente privados, tal y como establecen la Convención de la Unesco de 1970, el Convenio Unidroit de 1995 y, en el supuesto de los Estados miembros de la Unión Europea, la Directiva de 2014 reflejada también en el texto. Si bien el establecimiento de normas con contenido similar a estas, que solo cuentan con naturaleza política, prevendría a futuro la posesión de obras de dudosa procedencia, no resuelve los problemas derivados de las adquisiciones anteriores a él, realizadas sin haber comprobado la procedencia del bien o que, a pesar de haberlo hecho, debido al momento de su adquisición o a otras dificultades, no se pudo confirmar la licitud de su origen.

\section{Referencias}

Caamiña, C., "Inmunidad de jurisdicción y plazos de prescripción: caso Cassirer", en Anuario de la Facultad de Derecho de la UAM, 2015, 19, pp. $79-100$.

Forrest, C., "Immunity from seizure and suit in Australia: the protection of cultural objects on Loan Act 2013", en International Journal of Cultural Property, 2014, 21, (1), pp. 143-172.

Getz, D., "Canadian immunity from seizure legislation history", en International Journal of Cultural Property, 2011, 18, pp. 201-224. 
Graham, J., 'From Russia' without love: can the Shchukin heirs recover their ancestor's art collection?", en Sports and Entertainment Law Journal, 2009, pp. 66-107, en http://www.law.du.edu/documents/ sports-and-entertainment-law-journal/issues/06/From-RussiaWithout-Love.pdf

Jayme, E., "Globalization in art law", en V anderbilt Journal of Transnational Law, 2005, 38, pp. 927-945.

Kaye, L. M., "Art loans and immunity from seizure in the United States and the United Kingdom", en International Journal of Cultural Property, 2010, 17, pp. 335-359.

Knerly, S. J. Jr., Gest, K. L., Loeser, H. \& Parks, L. L. P., “International loans State immunity and anti-seizure laws", en ALI-ABA Course of Study Legal Issues in Museum Administration, 2009, Boston, Massachusetts, en http:/ / www.lending-for-europe.eu/fileadmin/CM/public/training/Antwerp/ALI-ABA_2009_Summary_of_Seizure_Laws.pdf

Kurtz, M. J., "The allied struggle over cultural restitution, 1942-1947”, en International Journal of Cultural Property, 2010, (17), pp. 177-194.

Merryman, J., "Two ways of thinking about cultural property", en American Journal of International Law, 1986, 80, pp. 831-853.

O’Connell, A., “The United Kingdom's immunity from seizure legislation", en Law, society, economy, working paper, en http:/ / eprints.lse. ac.uk/24583/1/WPS2008-20_OConnell.pdf

Palmer, N., "Adrift on a sea troubles: crossborder art loans and the specter of ulterior title", en Vanderbilt Journal of Transnational Law, 2005, 38, (4), pp. 947-996.

Pérez-Prat Durbán, L., "Las vicisitudes del patrimonio cultural: arte y derecho", en Curso de derecho internacional y relaciones internacionales de Vitoria-Gasteiz de 2006, Vitoria, pp. 241-273.

Pop, L., "Arresting art loan seizures", en Columbia-VLA Journal of Law and Arts, 2001, 24, (2), pp. 213-233.

Prott, L. V., "The Dja Dja Wurrung Bark etchings case”, en International Journal of Cultural Property, 2006, 13, (2), pp. 241-246.

Reinisch, A., "European Court prectice concerning State immunity from enforcement measures", en The European Journal of International Law, 2006, 17, pp. 803-816.

Rodríguez Pineau, E. \& Martínez Capdevila, C., "La protección de los bienes culturales en la UE: un régimen puesto a prueba”, en PérezPrat Durbán, L. \& Lazari, A. (coords.), El tráfico de bienes culturales, Tirant lo Blanch, Valencia, 2015, pp. 227-269. 
Symeonides, S. C., "A choice-of-law for conflicts involving stolen cultural property", en Vanderbilt Journal of Transnational Law, 2005, (38), pp. 1177-1198.

Van Woudenberg, N., "Immunity from seizure: which way leads to Rome?", actas de la conferencia dictada en Opening Session Collection Mobility 2.0., Museo Nacional del Prado, $31^{\text {st }}$ May- ${ }^{\text {st }}$ June 2010, Madrid, en http:/ / www.lending-for-europe.eu/fileadmin/CM/public/ training/madrid/van_Woudenberg-_Inmunity_from_seizure.pdf

Van Woudenberg, N., State immunity and cultural objects on loan, Martinus Nijhoff Publishers, Leiden-Boston, 2012.

Weller, M., "Immunity for artworks on loan? A review of international customary law and municipal anti-seizure statutes in light of the Liecbtenstein litigation", en Vanderbilt Journal of Transnational Law, 2005, 38, pp. 997-1039.

Weller, M., "The safeguarding of foreign cultural objects on loan in Germany", en Rivista di arte e diritto on line, 2009, (2), p. 1. 